REVIEW

\title{
Measuring quality of life
}

\author{
Christine Eiser, Meriel Jenney
}

Arch Dis Child 2007;92:348-350. doi: 10.1136/adc.2005.086405

Progress in the assessment of quality of life (QoL) measures for children and their value in clinical practice are considered. The most parsimonious approach to assessment includes modular assessment of generic and disease-specific QoL. Differences occur between child and parent proxy ratings, so it is recommended that ratings from multiple observers are made where possible. QoL measures may be of value in clinical practice, but the inadequate availability of the resources necessary to analyse results and provide the required support is a major barrier. Although the assessment of QoL in children remains challenging, its value is increasingly recognised by both researchers and clinicians.

See end of article for authors' affiliations

........................

Correspondence to: Dr Meriel Jenney, Children's Hospital for Wales, Heath Park, Cardiff CF1 4 4XW, UK; meriel. jenney@cardiffandvale. wales.nhs.uk

Accepted

30 November 2006
T e assessment of health-related quality of life (QoL) has been increasingly recognised as providing an important marker of health outcome for children in the general population and for those with chronic or life-threatening conditions. The impact of chronic conditions (eg asthma and diabetes) and their treatment can adversely affect the QoL of the child as a result of hospital appointments, restricted activities and general worry. For those with life-threatening conditions such as cancer, cure rates are often good, but there may be significant changes in QoL following treatment depending on the therapy used. In palliative care, the sole purpose of therapy may be to improve QoL. Given that traditional measures of mortality and morbidity are too narrow in these situations, there has been much discussion of the meaning and measurement of QoL, and recognition of the unique demands of measuring QoL in children compared with adults

Although there have been a number of previous reviews of the assessment of QoL in this journal, related journals ${ }^{2}$ and books, ${ }^{3}{ }^{4}$ we aim to consider achievements in the development of reliable and valid QoL measures, and how far they have been integrated into research or clinical practice.

\section{THE ASSESSMENT OF QOL}

\section{Measure development}

There has been a flood of new QoL measures, both generic (which can be used with different diagnostic patient groups and/or "normal" children and therefore facilitate comparisons across groups) and disease specific (which are assumed to have greater sensitivity to the implications of a single condition by including questions specific to the condition in question). Combining the merits of both approaches, a number of authors have developed generic measures with disease-specific modules. For example, this approach was used by Varni and colleagues for the PedsQL. ${ }^{5}$ Specific modules for assessment of children with asthma, arthritis and diabetes are now available, and further development for other conditions is in progress. A similar model was adopted by the DISABKIDS group, but this includes generic, chronic illness generic ${ }^{7}$ and disease-specific modules. ${ }^{8}$ The advantages of this modular approach are brevity and comprehensiveness. Independent disease-specific measures (eg cystic fibrosis ${ }^{9}$ and inflammatory bowel disease ${ }^{10}$ ) can provide more detailed assessment of QoL but lack provision for comparison with other groups.

The question of age-sensitivity has plagued the development of measures in children. On the one hand, the meaning of QoL changes with age and developmental level, suggesting that sensitive measures must address the issues of central concern for children of different ages. On the other hand, measures that target narrow age ranges are inadequate in situations, such as clinical trials, where the aim is to assess changes in QoL over time. Although most measures target school-aged children, ${ }^{11}$ a number have been developed for the very young ${ }^{12}$ and adolescents and young people. ${ }^{13}$

Chronic illness and treatment also impact on the wider family. In recognition of this, formal measures to assess the QoL of the care $\operatorname{giver}^{14}$ have been described and again are of potential value in clinical trials. Where treatments involve comparable levels of morbidity, the impact on the family and especially the burden on the primary care giver can realistically be taken into account. However, the care giver's own QoL can bias their reporting of the child's QoL (see below).

\section{Proxy rater issues}

For very young children and those of any age who are unable to meet the cognitive and communication demands involved, measures of QoL are dependent on proxy ratings of the child's QoL. The poor to moderate correlations that characterise parent and child responses to QoL assessments have challenged the value of the parent proxy as a straightforward substitute for the child's own rating. However, proxy ratings, particularly when used with the child's own reported QoL, can be used to gain multiple perspectives and thereby supply information over and above that provided by the patient alone. Explanations for the discrepancies have focused on characteristics of the patient, those of the proxy and the specific aspect of QoL being addressed. Children generally report less adverse impact on QoL and fewer problems than their parents or clinicians, but this varies with the child's health condition. 
Parent-child agreement is normally greater for sick compared with healthy children. Parents' reports appear to be more reliable (in that their ratings agree with the child's) for observable behaviours, such as physical symptoms and function, but less reliable for cognitive and emotional attributes. The evolving consensus is that multi-informant perspectives are preferable and supplement self-ratings of QoL but do not replace them. ${ }^{11}$

\section{The quality of measures}

The validity of any measure of QoL is clearly vital. It is important to know that we are measuring what we think we are measuring, but this is more complex than generally assumed. It is common to read that a measure is "valid" where the emphasis is on empirical validity or the extent to which the measure correlates with related phenomena. Much more complex is the idea of construct validity or the extent to which a measure reflects the variability among subjects on an underlying continuum. For example, how far do derived scores relate to the continuum of poor to excellent QoL?

Construct validity cannot be assumed because one measure correlates with another or because an appropriate underlying structure can be identified through factor analysis. A critical misassumption is that validity is a property of a measure; in fact, validity is only inferred from the assumptions about individuals that are drawn from the scores obtained. There is no gold standard. Misunderstandings about definitions of validity and the implications for measuring QoL continue to create a barrier to clinical and research use.

\section{Relationship between demographic and personality variables and QoL}

QoL has been distinguished from similar concepts such as health status or well-being but also needs to be distinguished from related psychological concepts such as happiness or optimism. In studies involving adults, one of the most important predictors of poor QoL is neuroticism. This is probably mediated by the tendency of those with high neuroticism to perseverate over their problems, focus on, recall and report more physical symptoms, and adopt less functional coping styles. Predictors of good QoL include extroversion and conscientiousness. Personality variables are closely related to how individuals perceive their own QoL $^{15}$ and also how they manage disability or illness, thus in turn influencing the different dimensions of their QoL.

\section{CHALLENGES TO QOL ASSESSMENT}

The development and availability of measures has prompted a wave of research aimed at describing the QoL of children with various health conditions. Although much of this work supports the basic assumption that QoL is compromised by chronic illness, some findings have proved difficult to explain. Sick children do not report as compromised a QoL as might be expected. ${ }^{16}$ Although their QoL scores may be compromised in the period immediately following diagnosis, in the longer term there is consistent evidence that children report QoL to be as good as, or sometimes better than, that of healthy controls. ${ }^{17}$ Attempts to explain this have focused on "response shift", the idea that hopes and aspirations are modified in line with changing health. These findings suggest that QoL is indeed a complex dynamic concept. Definitions of QoL may need to take into account individuals' resilience or ability to redefine their goals and ambitions in line with changing circumstances.

\section{Value of QoL measurement in clinical care}

There are arguments that inclusion of QoL measures in clinics is an unnecessary burden on patients and an intrusion into clinic time, but they have been used successfully with adults.
Self-reported QoL can guide decisions by providing information particularly relevant to the individual's clinical care which may otherwise not have been raised in the outpatient setting. However, it must be remembered that while assessment of QoL is possible, it is of little value if facilities or expertise for improvement are unavailable. Integration of QoL assessment into routine clinical practice therefore needs careful consideration. There is no point in assessing QoL if we are unable to change it.

One of the most serious barriers to making QoL assessment part of routine care may be the perceived costs of data collection and interpretation. It is likely that costs would increase in the short-term, but the hope is that by addressing patient problems early long-term costs would be reduced.

\section{THE FUTURE: WHAT PROGRESS?}

Although it was anticipated that the development of QoL measures would facilitate research, particularly in evaluations of clinical trials or different treatments, progress has been relatively slow. Problems relate to practical barriers, especially where multi-centre collaboration is required, as well as the limitations of current measures. These include a lack of sensitivity and responsiveness to change, the interpretation of QoL scores and the significance of numerical change of QoL, and a lack of understanding of psychometric theory and the meaning of different types of validity.

Despite the proliferation of disease-specific measures, some gaps are inevitable. While there have been a number of measures for children with relatively common conditions such as asthma, developing measures for children with rare conditions is complex and difficult. In these cases, researchers must rely on generic measures that lack sensitivity. Measures to assess QoL in palliative care are vital, as QoL is the only variable of real importance to families, children and clinicians. It is also important to reflect that the impact of disease and treatment on children is intimately related to the impact on the wider family. QoL assessment of the family, or at least the care giver, needs to be part of routine paediatric practice.

In terms of the sheer number of measures, the field has achieved considerable success. It is also true that the concept of QoL has much higher visibility in the paediatric field than even 10 years ago. QoL measures are being integrated into evaluations of clinical trials, partly through directives from funding bodies, although it will be some time before the results of such trials become available.

\section{Authors' affiliations \\ Christine Eiser, Department of Psychology, University of Sheffield, Sheffield, UK \\ Meriel Jenney, Children's Hospital for Wales, Cardiff, UK \\ Competing interests: None.}

\section{REFERENCES}

1 Jenney ME, Campbell S. Measuring quality of life. Arch Dis Child 1997;77:354

2 Eiser C, Jenney ME. Measuring symptomatic benefit and quality of life in paediatric oncology. Br J Cancer 1996;73:1313-16.

3 Eiser C. Children with cancer: the quality of life. Mahwah, NJ: Lawrence Erlbaum, 2004.

4 Koot HM, Wallander JL. Quality of life in child and adolescent illness: concepts, methods and findings. East Sussex, UK: Brunner-Routledge, 2001.

5 Varni JW, Seid M, Rode MA. The PedsQL: measurement model for the pediatric quality of life inventory. Med Care 1999;37:126-39.

6 Ravens-Sieberer U, Gosch A, Abel T, et al. Quality of life in children and adolescents: a European public health perspective. Soz Praventivmed $2001 ; 46: 294-302$

7 Peterson C, Schmidt S, Power M, et al. Development and pilot testing of a healthrelated quality of life chronic generic module for children and adolescents with chronic health conditions: a European perspective. Qual Life Res 2005; 14:1065-77 
8 Baars RM, Atherton $\mathrm{Cl}$, Koopman HM, et al. The European DISABKIDS project: development of seven condition-specific modules to measure health related quality of life in children and adolescents. Health Qual Life Outcomes 2005;3:70.

9 Quittner AL, Sweeney S, Watrous M, et al. Development of disease-specific quality of life measure for cystic fibrosis: linguistic validation of the French CFQ. J Pediatr Psychol 2000;25:403-14.

10 Otley A, Smith C, Nicholas D, et al. The IMPACT questionnaire: a valid measure of health-related quality of life in pediatric inflammatory bowel disease. J Pediatr Gastroenterol Nutr 2002;35:557-63.

11 Eiser C, Morse R. Quality-of-life measures in chronic diseases of childhood. Health Technol Assess 2001;5:1-157.

12 Christie MJ, French D, Sowden A, et al. Development of childcentred diseasespecific questionnaires for living with asthma. Psychosom Med 1993;55:514-8.
13 Bhatia S, Jenney ME, Bogue MK, et al. The Minneapolis-Manchester Quality of Life instrument: reliability and validity of the adolescent form. J Clin Oncol 2002;29:4692-8

14 Juniper EF, Guyatt GH, Feeney GH, et al. Measuring quality of life in the parents of children with asthma. Qual Life Res 1996:5:27-34.

15 Vollrath M, Landolt MA. Personality predicts quality of life in pediatric patients with unintentional injuries: a 1-year follow-up study. J Pediatr Psychol 2005;30:481-91.

16 De Clercq B, De Fruyt F, Koot HM, et al. Quality of life in children surviving cancer: a personality and multi-informant perspective. J Pediatr Psychol 2004; 29:579-90.

17 Zebrack BJ, Chesler MA. Quality of life in childhood cancer survivors. Psychooncology 2002;11:132-41.

\section{ARCHIVIST}

\section{Dilated cardiomyopathy}

$\mathrm{T}$

he World Health Organization classification of cardiomyopathies has four categories: dilated cardiomyopathy, hypertrophic cardiomyopathy, restrictive cardiomyopathy, and arrhythmogenic right ventricular dysplasia-cardiomyopathy. Although there may be some overlap, most patients are classifiable as having one of these types. Dilated cardiomyopathy (DCM), with a dilated left ventricle and systolic dysfunction, is the most common form and the most common reason for heart transplantation in children and adults. Incidences of 0.34 and 1.13 cases per 100000 children per year have been reported from Finland and the USA. Now, a large epidemiological study has been reported from the US (Jeffrey A Twobin and colleagues. JAMA 2006;296:1867-76).

The study included two prospective cohorts in New England and the central Southwestern USA and a retrospective cohort based on 39 tertiary centres in the USA and Canada. In all, there were 1426 children with DCM. The annual incidence of DCM in children $<18$ years of age was 0.57 cases per 100000 children per year. The incidence was higher in boys than in girls ( $0.66 \mathrm{vs}$ 0.47 cases per 100000 per year), in black children than in white (0.98 vs 0.46$)$, and in infants than in older children (4.4 vs 0.34 ). Two thirds of children (66\%) had idiopathic DCM, 16\% had myocarditis, 9\% neuromuscular disorders (usually Duchenne or Becker muscular dystrophy), 5\% familial DCM, 4\% inborn errors of metabolism and $1 \%$ malformation syndromes. Of the 66 cases of familial DCM, 45 had autosomal dominant inheritance, 16 autosomal recessive, $1 \mathrm{X}$ linked and 4 the complex phenotype of left ventricular non-compaction with gene mapping. The main types of inborn errors of metabolism ( 54 cases) were mitochondrial disorders $(n=20)$, Barth syndrome $(n=13)$, and primary or systemic carnitine deficiency $(n=7)$. Of the 15 patients with malformation syndromes, there were 7 with a chromosomal defect and 5 with Alström syndrome.

The 1-year and 5-year survival rates after the diagnosis were $87 \%$ and $77 \%$, respectively. The corresponding rates of survival without transplantation were $69 \%$ and $54 \%$. The median age at diagnosis was 1.5 years, at decision to transplant 4 years, at transplantation 4.8 years and at death (among those who died during the study) 3 years. A quarter of the survivors had been followed up for $>4$ years. Risk factors for death or transplantation present at diagnosis were older age, congestive heart failure, lower left ventricular fractional shortening z score and cause of DCM (risk doubled with idiopathic DCM compared with myocarditis).

The incidence of DCM varies with age, sex and race, and prognosis depends on the cause, the age of the child at diagnosis, and the presence or absence of congestive heart failure. 